\title{
Association of polymorphism of PTPN 11 encoding SHP-2 with gastric atrophy but not gastric cancer in Helicobacter pylori seropositive Chinese population
}

Jing Jiang ${ }^{1}$, Zhi-Fang Jia', Fei Kong ${ }^{1}$, Mei-Shan Jin², Yin-Ping Wang ${ }^{2}$, Suyan Tian ${ }^{1}$, Jian Suo ${ }^{3}$ and Xueyuan Cao ${ }^{3 *}$

\begin{abstract}
Background: The interaction between Src homology 2 domain-containing protein tyrosine phosphatase (SHP-2) of gastric epithelial cells and cagA from H. pylori plays a crucial role in developments of gastric atrophy and gastric cancer. This study aimed to investigate the association of haplotype tagging SNPs (htSNPs) in the PTPN11 gene encoding SHP-2 with gastric atrophy and gastric cancer in Chinese population.

Methods: The subjects comprised 414 patients with gastric cancer, 109 individuals with gastric atrophy and 923 healthy controls. Blood was collected from October 2008 to October 2010. Five htSNPs rs2301756, rs12423190, rs 12229892, rs7958372 and rs4767860 from the PTPN11 gene were selected and genotyped by Taqman assay. Serum Ig $G$ antibodies to H. pylori were detected by ELISA. Gastric atrophy was screened by the levels of serum pepsinogenlandll, and confirmed by endoscopy and histopatholgical examinations. Odds ratio (ORs) and 95\% confidence intervals $(\mathrm{Cls})$ were calculated by a multivariate logistic regression.

Results: Among H. pylori seropositive subjects, age and gender-adjusted OR of gastric atrophy was $2.47(95 \% \mathrm{Cl}$ 1.13-4.55, $P=0.02$ ) for $C C$ genotype compared with $C T / T T$ genotypes, suggesting a recessive model of genetic risk for rs 12423190 . The prevalence of $\mathrm{H}$. pylori seropositivity were significantly higher in groups of gastric cancer and gastric atrophy compared to the control group ( $70.3 \%$ vs. $75.2 \%$ vs. $49.7 \%, P<0.001)$. However, the distributions of genotypes and haplotypes in patients with gastric cancer were not significantly different from healthy controls.

Conclusions: Our study provides the first evidence that rs 12423190 polymorphism of the PTPN11 gene is significantly associated with an increased risk of gastric atrophy in H. pylori infected Chinese Han population, suggesting that rs 12423190 polymorphism could be used as a useful marker of genetic susceptibility to gastric atrophy among $H$. pylori infected subjects. The biological roles of this polymorphism require a further investigation.
\end{abstract}

\section{Background}

Gastric cancer is the most common malignancy of gastrointestinal tract in East Asian populations and the third most common cause of cancer-related deaths in China [1,2]. Helicobacter pylori (H. pylori) infection has been established as a major risk factor for gastric cancer, through the induction of gastric atrophy and progression of precancerous lesions by numerous studies [3,4]. Although $H$. pylori is estimated to inhabit at least half of

\footnotetext{
* Correspondence: ccmzc32jdyycao@yahoo.com.cn

${ }^{3}$ Department of Gastric and Colorectal Surgery, Jilin University First Hospital,

Changchun, China

Full list of author information is available at the end of the article
}

the world's human population, just few subjects develop to gastric precancerous lesions and adenocarcinoma. The extent of gastric damages induced by H. pylori infection seems to vary from one subject to another, suggesting that the combination of host genetic traits and bacterial virulence plays important roles in long-term outcomes of $H$. pylori infection [5-7].

Several studies have provided evidences that infection with cagA-positive $H$. pylori associates with higher grades of gastric inflammation and is more virulent than the cagA-negative strains [8]. The CagA protein is delivered into gastric epithelial cells via the bacterial type IV secretion system, where it undergoes tyrosine phosphorylation

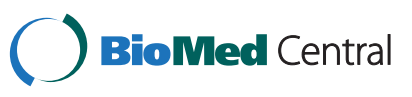


by Src and Abl kinases. Tyrosine-phosphorylated CagA then acquires capability to interact with and deregulate SHP-2 phosphatase, a bona-fide oncoprotein [9]. The formation of cagA/SHP-2 complex induces abnormal proliferation and migration of gastric epithelial cells, consequently resulting in gastric atrophy and gastric carcinoma [10-12]. In addition, gain-of-function mutations of the SHP-2 have recently been found in human malignancies [13-15]. Kim et al also revealed that gastric cancers displayed higher levels of SHP-2 protein compared to normal cells, suggesting that neo-expression of this signalling protein in cells might play a role in the gastric carcinogenesis [16]. Since the protein-tyrosine phosphatase nonreceptor-type 11 (PTPN11) gene encodes protein containing two tandem Src homology-2 domains, which function as phospho-tyrosine binding domains. SHP-2 closely interacts with the CagA protein, therefore, it is speculated that functional polymorphisms in the PTPN11 may mediate the interaction of this protein with its substrates and affect its regulatory role in various cell signalling events, such as mitogenic activation, metabolic control, transcription regulation, cell migration, and malignant transformation in H. pylori infected subjects.

The PTPN11 gene is on chromosome 12, containing 16 exons. Several single-nucleotide polymorphisms (SNPs) rs11066322, rs11066320 and rs2301756 have been identified in Caucasian females to be associated with apoB levels and LDL-C levels [17]. Another study demonstrated that the rs11066322 was associated with increased plasma HDL-C levels [18]. These results suggested that genetic variants influencing SHP-2 activities may modulate biological functions of the protein. In gastric cancer, Japanese group has found that a prevalent SNP in intron3 (rs2301756) was associated with an increased risk of gastric atrophy in Japanese population with $H$. pylori infection [19-22]. The aim of the present study is to determine whether polymorphisms of PTPN11 gene are associated with clinical outcomes of $H$. pylori infection in Chinese population.

\section{Methods}

\section{Study populations}

Four hundred and fourteen Gastric cancer cases were selected from the department of gastric and colorectal surgery, the First Hospital, Jilin University, from 2008 to 2010. All patients underwent tumor resection with histologically confirmed diagnosis of gastric adenocarcinoma. The gastric atrophy individuals and health controls were recruited from the healthy check-up centre of the same hospital from 2009 to 2010. A total 1080 persons (630 males and 450 females, aged 35 to 80 years old) participated in the study without history of cancer. The examinees were Han inhabitants in Changchun city. The informed consent was obtained from all subjects and the study protocol was approved by the ethics committee of the first affiliated hospital, Jilin University. The examinees received serum anti- $H$. pylori IgG titre and pepsinogen examinations for screening $H$. pylori infection and gastric atrophy.

\section{Tests for H. pylori infection and diagnosis of gastric Atrophy}

Serum immunoglobulin (Ig) G antibodies to H. pylori were detected by enzyme-linked immunosorbent assay (ELISA) using H. pylori -IgG ELISA kit (Biohit, Helsink, Finland). The antibody titres were defined by optical density (OD) values according to the manufacturer's protocol and titres higher than the cut off value of 30EIU, were considered as positive for $H$. pylori infection. PepsinogenIand II (PGIand PGII) in serum were measured using ELISA kits (Biohit, Helsink, Finland). For screening gastric atrophy, we decided to use cut-off points of $<82.3 \mathrm{ng} / \mathrm{ml}$ for PGIand $<6.05$ for PGI/PGIIratio due to no universally accepted cut-off points for dichotomising PGI or the PGI/PGII ratio, which have been validated against histological confirmatory studies for gastric atrophy [23]. The kit quality control samples showed Coefficient of variations (CVs) of 4.5, 4.3 and 4.7\% for H. pylori, PGI and PGII, respectively. All suspected cases with gastric atrophy by serum screening were re-determined by endoscopic, biopsy and histological examinations for a definite diagnosis.

\section{Selection of tagging SNPs}

The principal hypothesis underlying this experiment is that one or more common SNPs in PTPN11 gene are associated with an altered risk of gastric atrophy and/or gastric cancer. Thus, the aim of the SNP tagging is to identify a set of SNPs that efficiently tags all known SNPs. We postulate that such SNPs are also likely to tag any hitherto unidentified SNPs in PTPN11 gene. Haplotype tagging SNPs (htSNPs) were selected from the Han Chinese data in the HapMap Project (06-02-2009 HapMap) using the SNPbrowser ${ }^{\text {Tw }}$ Software v4.0 to capture SNPs with a minimum minor allele frequency (MAF) of 0.05 with a pair-wise $r$ square of 0.8 or greater [24]. There are 9 SNPs at minor allele frequencies $>0.05$ in the PTPN11 gene in Chinese on HapMap, all of which are located in noncoding regions of the PTPN11 gene (Figure 1). Finally, five htSNPs rs2301756, rs12423190, rs12229892, rs7958372 and rs4767860 were selected and four of them are shown to be in a complete linkage disequilibrium (LD) with Tag SNP $\left(\mathrm{D}^{\prime}=1\right.$ and $\left.\mathrm{r}^{2}>0.8\right)$.

\section{Genotyping}

Genomic DNA from whole blood was extracted with an AxyPrep blood Genomic DNA extraction Kit (AP-MNBL-GDNA-250, Axygen Biosciences, Union City, USA). Polymerase chain reactions (PCR) were carried out on 


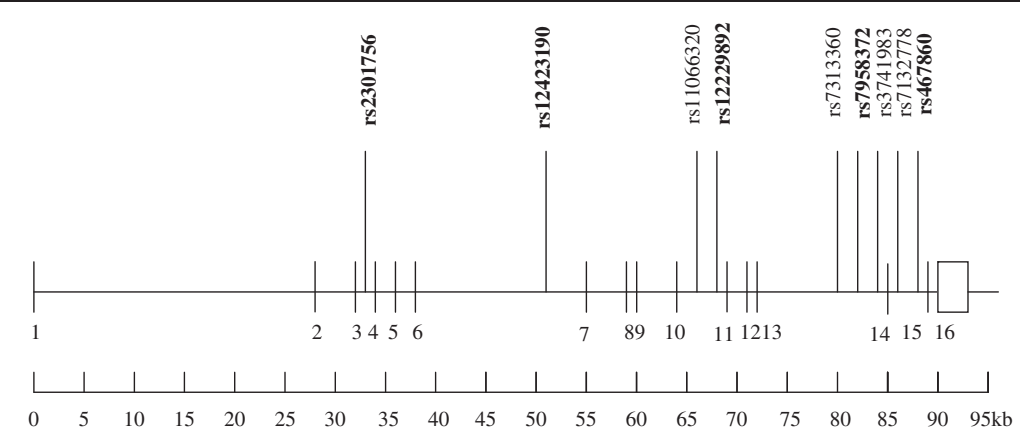

Figure 1 Genomic map of PTPN11 gene. Validated SNPs are illustrated and htSNPs genotyped in the present study are indicated in bold. Vertical Lines and a box represent exons.

genomic DNA (10 ng) using a TaqMan universal PCR master mix (Applied Biosystems). Forward and reverse primers and FAM and VIC labelled probes were designed by Applied Bio systems (ABI Assay-by-Designs) in a 5 ul reaction. Sequences of primers and probes are available on request. Amplification conditions on BIO-RAD S1000 thermal cyclers (Bio-Rad Laboratories, Hercules, California) were as follows: 1 cycle of $95^{\circ} \mathrm{C}$ for $10 \mathrm{~min}$, followed by 40 cycles of $95^{\circ} \mathrm{C}$ for $15 \mathrm{~s}$ and $60^{\circ} \mathrm{C}$ for $1 \mathrm{~min}$. The completed PCR products were read on an ABI PRISM 7900 Sequence Detector in end-point mode using the Allelic Discrimination Sequence Detector Software V2.3 (Applied Biosystems). For the software to recognize genotypes, two non-template controls were included in each 384-well plate. All patients' and normal control samples were arrayed together in four 384-well plates and the fifth plate contained eight duplicate samples from each of four plates to ensure the quality of genotyping (the concordance was $>99 \%$ for all SNPs).

\section{Statistics analysis}

For each polymorphism, the deviation of genotype frequencies in controls from those expected under HardyWeinberg equilibrium was assessed by a goodness-of-fit $\mathrm{x}^{2-}$ test. Linkage disequilibrium (LD) between pairs of biallelic loci was determined using two measures, D' and $r^{2}$. Either Chi-square test or Fisher's exact test was performed by comparing distributions of genotype frequencies between patients and controls. Risks associated with rare genotypes were estimated as odds ratios (ORs). Corresponding 95\% confidence intervals (CIs) by an unconditional logistic regression were adjusted by age (scale variable) and sex (nominal variable). For haplotypes with frequencies $>1 \%$, risks were compared to the reference haplotype(major haplotype in control group) using an unconditional logistic regression model with the HAPSTAT 3.0 software, according to Lin et al [Copyright (c) 2006-2008 Tammy Bailey, Danyu Lin and the University of North Carolina, NC, USA.] [25,26]. HAPSTAT allows the user to estimate or test haplotype effects and haplotype-environment interactions by maximum likelihood estimation and the EM algorithm. All statistical tests were two-tailed and $P$-values were considered to be statistically significant when $\leqq 0.05$. All analyses were performed using a statistical analysis software for windows, version 9.2 (SAS Institute, Cary, NC, USA). The statistical power calculations were performed using the QUANTO software program (Version 1.2.3).

\section{Results}

Characteristics of subject allele frequencies of the htSNPs

A total of 414 patients gastric cancer (300 males and 114 females) aged 35 to 80 years old were included in this study. Of 1080 examinees, 148 individuals were screened for gastric atrophy using serum PG examination; 109 patients were confirmed with gastric atrophy by biopsy and histopathological examinations; 17 people were diagnosed as pseudopositive for gastric atrophy and excluded from the study; 22 people who rejected endoscopic examination were excluded from the study. Nine hundred and thirty-two individuals were included in the control group. Nine persons were excluded, due to the failure to detected anti-H. pylori Ig G and/or genotyps in blood samples. Finally, 923 subjects were included and analysed as control. The characteristics of subjects are summarized in Table 1. The mean age was older in gastric cancer patients than that in controls $(61.5$ vs. 50.7 years; $P<0.001)$. There were more females in the control group $(P<0.001)$. Prevalence of $H$. pylori seropositivity were significantly higher in groups of gastric cancer and gastric atrophy than that in the healthy control $(70.3 \%$ vs. $49.7 \%, P<0.001 ; 75.2 \%$ vs. 49.7\%, $P=0.01$ ).

The linkage disequilibrium structure of the 5 polymorphic loci is shown in Figure 2. All D' values are above 0.93, and the pair-wise comparison among rs12423190 and rs12229892 revealed a complete LDs $\left(\left|D^{\prime}\right|=1\right.$ and $\left.r^{2}=0.285\right)$. The genotype distribution for five htSNPs in the control group were in Hardy-Weinberg equilibrium $(P$ value were $0.54,0.94,0.78,0.68$ and 0.87 respectively). In the present study, the distribution 
Table 1 Characteristics of the subjects and the PTPN11 polymorphisms

\begin{tabular}{|c|c|c|c|c|}
\hline & $\begin{array}{l}\text { Gastric cancer } \\
(\%)\end{array}$ & $\begin{array}{l}\text { Gastric atrophy } \\
(\%)\end{array}$ & $\begin{array}{l}\text { Control } \\
(\%)\end{array}$ & $P$ value \\
\hline $\mathrm{N}$ & 414 & 109 & 923 & \\
\hline \multicolumn{5}{|l|}{ Sex } \\
\hline Male & $300(72.5)$ & $64(58.7)$ & $539(58.4)$ & \multirow[t]{2}{*}{$<0.001$} \\
\hline Female & $114(27.5)$ & $45(41.3)$ & $384(41.6)$ & \\
\hline \multicolumn{5}{|l|}{ Age } \\
\hline$\leq 45$ & $32(7.7)$ & 19(17.4) & 276(29.9) & \multirow[t]{3}{*}{$<0.001$} \\
\hline $46-65$ & 224(54.1) & $80(73.4)$ & $579(62.7)$ & \\
\hline$>65$ & 158(38.2) & $10(9.2)$ & $68(7.4)$ & \\
\hline \multicolumn{5}{|c|}{ Anti-H.pylori lgG } \\
\hline Negative & 123(29.7) & $27(24.8)$ & $464(50.3)$ & \multirow[t]{2}{*}{$<0.001$} \\
\hline Postive & 291(70.3) & $82(75.2)$ & $459(49.7)$ & \\
\hline \multicolumn{5}{|l|}{ SNPS } \\
\hline \multicolumn{5}{|l|}{ rs2301756 } \\
\hline GG & $305(73.7)$ & 86(78.9) & $687(74.4)$ & \multirow[t]{3}{*}{0.462} \\
\hline GA & $95(22.9)$ & $22(20.2)$ & $216(23.4)$ & \\
\hline $\mathrm{AA}$ & $14(3.4)$ & $1(0.9)$ & $20(2.2)$ & \\
\hline \multicolumn{5}{|c|}{ rs12423190 } \\
\hline$\pi$ & 214(51.7) & $55(50.5)$ & $476(51.6)$ & \multirow[t]{3}{*}{0.047} \\
\hline $\mathrm{TC}$ & 166(40.1) & $36(33.0)$ & $373(40.4)$ & \\
\hline $\mathrm{CC}$ & $34(8.2)$ & 18(16.5) & $74(8.0)$ & \\
\hline \multicolumn{5}{|c|}{ rs12229892 } \\
\hline GG & 137(33.1) & $40(36.7)$ & 308(33.4) & \multirow[t]{3}{*}{0.963} \\
\hline GA & 205(49.5) & $50(45.9)$ & $454(49.2)$ & \\
\hline AA & $72(17.4)$ & 19(17.4) & 161(17.4) & \\
\hline \multicolumn{5}{|c|}{ rs7958372 } \\
\hline$\pi$ & $305(73.7)$ & 87(79.8) & $685(74.2)$ & \multirow[t]{3}{*}{0.747} \\
\hline $\mathrm{TC}$ & 102(24.6) & 21(19.3) & $222(24.1)$ & \\
\hline $\mathrm{CC}$ & $7(1.7)$ & $1(0.9)$ & $16(1.7)$ & \\
\hline \multicolumn{5}{|c|}{ rs4767860 } \\
\hline AA & 137(33.1) & 39(35.8) & $306(33.2)$ & \multirow[t]{3}{*}{0.386} \\
\hline$A G$ & 206(49.8) & $44(40.4)$ & $453(49.1)$ & \\
\hline GG & $71(17.1)$ & $26(23.9)$ & 164(17.8) & \\
\hline
\end{tabular}

of rs12423190 genotype was found to be statistically different between groups $(P=0.047)$, however, distributions of rest four SNPs were not significantly different between groups.

Association between htSNPs and H. pylori seropositivity, gastric atrophy and gastric cancer

The age and sex-adjusted ORs of gastric atrophy among H. pylori seropositive subjects were $0.93(95 \% \mathrm{CI} 0.55$ 1.57, $P=0.78)$ for $\mathrm{CT}$ and 2.20 (95\%CI $1.06-4.55$, $P=0.03$ ) for CC genotype compared with rs 12423190

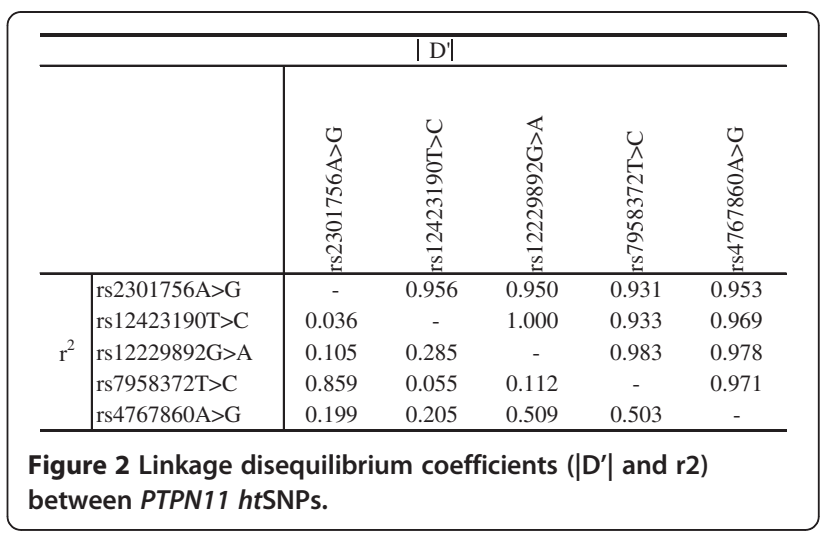

TT genotype. In a recessive model, the age- and sexadjusted OR was 2.47 (95\%CI 1.13-4.55, $P=0.02)$ for CC genotype versus rs12423190 CT/TT genotypes (Table 2). However, the age and sex-adjusted ORs of gastric cancer among $H$. pylori seropositive subjects was $1.18(95 \% \mathrm{CI}$ 0.64-2.18, $P=0.60$ ) for CT and 1.30 (95\%CI 0.93-1.83, $P=0.13)$ for CC genotype compared with rs12423190 TT genotype. The haplotypes with frequencies $\geq 1 \%$ are shown in Table 2. The most frequent haplotypes were GTATA (rs2301756G, rs12423190T, rs12229892A, rs7958372T, rs4767860A) and four major haplotypes were accounted for over $95 \%$ of distribution. There was no significant association between haplotypes and gastric atrophy. The OR of GCGTG haplotype was 1.36 (95\%CI $0.92-2.02, P=0.12$ ) versus the GTATA haplotype (Table 2). The association between the SNPs of PTPN11 and the H. pylori infection was examined in health controls (Table 3), but no significant association between five htSNPs of PTPN11 and $H$. pylori seropositivity was found. Distributions of PTPN11 haplotypes were not correlated with $H$. pylori seropositivity. No association between htSNPs and gastric atrophy in $H$. pylori seronegative subjects was found either (data not shown). In a subgroup analysis, we did not find any association of htSNPs with Lauren's classification, tissue differentiation and TNM staging (data not shown).

\section{Discussions}

CagA-secreting H. pylori infection plays an important role in gastric carcinogenesis via a sequential CagA signal transduction pathway. CagA initially binds to seven protein components to activate aberrant cellular responses that promote the development of gastric cancer. Since the function of CagA protein is regulated by its binding partners, therefore genes that encode CagA interacting molecules may modify the risk of gastric cancer. In the present study, 5 htSNPs of the PTPN11 gene were investigated for their associations with $H$. pylori infection, gastric atrophy and gastric cancer in Chinese Hans population. We found that subjects bearing 
Table 2 PTPN11 polymorphisms among $H$. pylori (+) subjects

\begin{tabular}{|c|c|c|c|c|c|c|c|}
\hline Genotype & $\begin{array}{l}\text { Control (\%) } \\
n=459\end{array}$ & $\begin{array}{l}\text { Gastric atrophy (\%) } \\
\mathrm{n}=82\end{array}$ & OR(95\%Cl) & $P$ value & $\begin{array}{l}\text { Gastric cancer (\%) } \\
\mathrm{n}=291\end{array}$ & OR(95\%Cl) $)^{*}$ & $P$ value \\
\hline \multicolumn{8}{|l|}{ rs2301756 } \\
\hline GG & 74.3 & 76.8 & Reference & & 73.9 & Reference & \\
\hline GA & 23.5 & 22.0 & $0.90(0.51-1.59)$ & 0.72 & 37.9 & $0.92(0.62-1.35)$ & 0.66 \\
\hline $\mathrm{A} / \mathrm{A}$ & 2.2 & 1.2 & $0.51(0.06-4.09)$ & 0.53 & 3.4 & $1.26(0.47-3.37)$ & 0.65 \\
\hline \multicolumn{8}{|l|}{ rs12423190 } \\
\hline$\pi$ & 52.5 & 50.0 & Reference & & 50.9 & Reference & \\
\hline TC & 40.1 & 34.1 & $0.93(0.55-1.57)$ & 0.78 & 41.2 & $1.18(0.64-2.18)$ & 0.60 \\
\hline $\mathrm{CC}$ & 7.4 & 15.9 & $2.20(1.06-4.55)$ & 0.03 & 7.9 & $1.30(0.93-1.83)$ & 0.13 \\
\hline Tा/TC & 92.6 & 84.1 & Reference & & 92.1 & Reference & \\
\hline CC & 7.4 & 15.9 & $2.47(1.13-4.55)$ & 0.02 & 7.9 & $1.21(0.89-1.76)$ & 0.15 \\
\hline \multicolumn{8}{|l|}{ rs12229892 } \\
\hline GG & 32.2 & 36.6 & Reference & & 30.6 & Reference & \\
\hline GA & 51.2 & 47.6 & $0.82(0.49-1.39)$ & 0.46 & 52.2 & $1.04(0.72-1.51)$ & 0.82 \\
\hline $\mathrm{AA}$ & 16.6 & 15.9 & $0.86(0.42-1.75)$ & 0.67 & 17.2 & $1.02(0.63-1.67)$ & 0.93 \\
\hline \multicolumn{8}{|l|}{ rs7958372 } \\
\hline$\pi$ & 74.1 & 78.0 & Reference & & 74.2 & Reference & \\
\hline TC & 24.4 & 20.7 & $0.80(0.45-1.43)$ & 0.46 & 24.1 & $0.94(0.64-1.37)$ & 0.74 \\
\hline $\mathrm{CC}$ & 1.5 & 1.2 & $0.83(0.10-7.01)$ & 0.86 & 1.7 & $1.16(0.30-4047)$ & 0.83 \\
\hline \multicolumn{8}{|l|}{ rs4767860 } \\
\hline AA & 33.8 & 34.1 & Reference & & 31.9 & Reference & \\
\hline$A G$ & 49.5 & 40.2 & $1.54(0.82-2.91)$ & 0.18 & 51.9 & $1.04(0.70-1.88)$ & 0.59 \\
\hline GG & 16.8 & 25.6 & $0.80(0.46-1.38)$ & 0.43 & 16.2 & $1.32(0.92-1.90)$ & 0.14 \\
\hline \multicolumn{8}{|l|}{ Haplotpe } \\
\hline GTATA & 41.3 & 39.6 & Reference & & 41.2 & Reference & \\
\hline GCGTG & 26.0 & 32.3 & $1.36(0.92-2.02)$ & 0.12 & 27.6 & $1.12(0.80-1.57)$ & 0.51 \\
\hline GTGTA & 16.0 & 14.0 & $0.96(0.58-1.61)$ & 0.89 & 14.4 & $1.14(0.88-1.47)$ & 0.32 \\
\hline ATGCG & 12.4 & 11.6 & 1.03(0.59-1.78) & 0.92 & 13.1 & $0.96(0.71-1.32)$ & 0.82 \\
\hline Others & 4.3 & 2.5 & & & 3.7 & & \\
\hline
\end{tabular}

"OR for each genotype and hapolotype were calculated by age and sex adjusted logistic regression model.

rs12423190 CC genotype at intron 6 had a significantly higher risk in $H$. pylori-seropositive gastric atrophy. The OR for gastric cancer was increased in those carrying the GCGTG haplotype versus the GTATA (OR $=1.30$, 95\%CI: 0.93-1.83), however, the $P$ value was 0.09 , not statistically significant. In this study, the allele frequencies of rs12423190 were $71.8 \%$ for the $\mathrm{T}$ allele and $28.2 \%$ for the $\mathrm{C}$ allele among control subjects. The frequency of the $\mathrm{C}$ allele was similar to that reported in the HapMap Project (31.4\%). Compared to the NCBI SNP database, we found that the frequency of the $C$ allele is obviously higher in Chinese population than other populations (23.3\% in Japanese; $11.5 \%$ in Utah residents with Northern and Western European ancestry). As incidences of gastric cancer are high in Chinese, Japanese and Koreans, high frequencies of this polymorphism in Asian populations may be part of explanation. Our findings indicated that the $\mathrm{C}$ allele contributes to genetic predisposition to $H$. pylori-induced gastric atrophy in Chinese population. A further study is required to confirm associations of the PTPN11 rs12423190 polymorphism with gastric atrophy in diverse ethnic populations.

Recent studies revealed significant associations between the rs2301756 AA genotype in intron3 and reduced risk of gastric atrophy among $H$. pylori-seropositive Japanese subjects $[7,19,22]$. Our study found a similar trend of decreased risk of OR for rs2301756, however, the OR of atrophy was not significantly lower compared with the GA genotype $(\mathrm{OR}=0.90,95 \% \mathrm{CI}$ : 0.51-1.59) and AA genotype ( $\mathrm{OR}=0.51,95 \% \mathrm{CI}$ : 0.06-4.09). In controversy, the association study for rs2301756 and gastric atrophy showed a completely opposite result 


\begin{tabular}{|c|c|c|c|c|}
\hline Genotype & $\begin{array}{l}\mathrm{Hp}+(\%) \\
(\mathrm{n}=459)\end{array}$ & $\begin{array}{l}\mathrm{Hp}-(\%) \\
(\mathrm{n}=464)\end{array}$ & OR $(95 \% \mathrm{Cl})^{*}$ & $P$ value \\
\hline \multicolumn{5}{|l|}{ rs2301756 } \\
\hline GG & 74.7 & 75.2 & Reference & \\
\hline GA & 23.3 & 22.8 & $1.18(0.51-2.72)$ & 0.71 \\
\hline AA & 2.0 & 2.0 & $1.52(0.49-4.66)$ & 0.47 \\
\hline \multicolumn{5}{|l|}{ rs12423190 } \\
\hline $\mathrm{T} / \mathrm{T}$ & 52.1 & 50.7 & Reference & \\
\hline $\mathrm{T} / \mathrm{C}$ & 39.2 & 40.1 & 0.98(0.55-1.76) & 0.95 \\
\hline$C / C$ & 8.7 & 9.2 & $0.92(0.29-2.93)$ & 0.89 \\
\hline \multicolumn{5}{|l|}{ rs12229892 } \\
\hline $\mathrm{G} / \mathrm{G}$ & 32.9 & 34.6 & Reference & \\
\hline $\mathrm{G} / \mathrm{A}$ & 50.6 & 46.8 & $1.25(0.93-1.70)$ & 0.15 \\
\hline $\mathrm{A} / \mathrm{A}$ & 16.5 & 18.5 & $1.01(0.65-1.57)$ & 0.97 \\
\hline \multicolumn{5}{|l|}{ rs7958372 } \\
\hline $\mathrm{T} / \mathrm{T}$ & 74.7 & 74.9 & Reference & \\
\hline $\mathrm{T} / \mathrm{C}$ & 23.8 & 23.2 & 0.84(0.40-1.77) & 0.65 \\
\hline$C / C$ & 1.5 & 1.8 & $0.61(0.13-2.79)$ & 0.53 \\
\hline \multicolumn{5}{|l|}{ rs4767860 } \\
\hline A/A & 33.8 & 33.0 & Reference & \\
\hline$A / G$ & 48.1 & 48.3 & $1.11(0.36-3.45)$ & 0.86 \\
\hline $\mathrm{G} / \mathrm{G}$ & 18.1 & 18.7 & $1.00(0.55-1.84)$ & 0.99 \\
\hline \multicolumn{5}{|l|}{ Haplotpe } \\
\hline GTATA & 41.0 & 41.6 & Reference & \\
\hline GCGTG & 26.9 & 28.4 & $0.97(0.79-1.20)$ & 0.77 \\
\hline GTGTA & 15.7 & 14.8 & 1.08(0.83-1.40) & 0.56 \\
\hline ATGCG & 12.3 & 13.0 & 0.94(0.71-1.24) & 0.66 \\
\hline Others & 4.1 & 2.2 & & \\
\hline
\end{tabular}

"OR for each genotype and hapolotype were calculated by age and sex adjusted logistic regression model.

in Uzbekistan population [20]. Zhu F and colleagues also demonstrated that the rs2301756 A allele was associated with low risk of intestinal metastasis (IM) indicating that H. pylori infection induces gastric precancerous lesions, such as gastric atrophy (OR $=0.46,95 \% \mathrm{CI}$ : 0.21-0.99) in H. pylori seropositive individuals. Meantime, an inverse association was shown in $H$. pylori-seronegative subjects in the same study $(\mathrm{OR}=2.51,95 \% \mathrm{CI}$ : 1.21-4.43) [27]. There were no statistically differences in the frequency of rs2301756 in our study and others [21,28]. Inconsistent results from different studies may be due to different environmental backgrounds and ethnic groups. In our study, gastric atrophy was only identified in 82 cases of $H$. pylori- seropositive subjects, the minor allele of rs2301756 was relatively rare $(\mathrm{MAF}=0.139)$. The statistical power maybe not sufficient $($ power $=0.10)$ to examine the association. A further investigation of association between rs 2301756 and gastric atrophy is needed in a large population and other ethnic groups.

The functions of PTPN11 polymorphisms are still unknown. SHP-2 contains two tandem SH2 domains, a PTP domain, and other functional motifs. Genetic mutations in PTPN11 exon 3(encoding the N-SH2 domain), exon 4(encoding the C-SH2 domain), Exons 7, 8 and 12 (encoding the PTP domain) have been identified in Noonan syndrome, juvenile myelomonocytic leukemia, LEOPARD syndrome, lung cancer, liver cancer and colon cancer [29]. The interaction of tyrosine phosphorylated Cag-A with the $\mathrm{SH} 2$ domain of the protein is supposed to induce a conformational change in SHP-2 that weakens the inhibitory interaction between PTP and N-SH2 domain, and results in activation of SHP-2's catalytic activity [10,30]. The rs12423190 polymorphism is located in the intron 6, 1408 bp upstream exon 7, encoding part of PTP domain. Using a free bioinformatic tool (http://fastsnp. ibms.sinica.edu.tw/pages/inputCandidateGeneSearch.jsp) the rs12423190 is predicted to locate in the side of an intronic enhancer which could affect the gene transcriptional regulation. SHP-2 has several biological functions. The gain-of-function of SHP-2 may accelerate the downregulation of $\mathrm{T}$-cell and B-cell activation through CTLA-4/PD-1 as well as IL-6/STAT3 signalings, eventually leading to a decrease in inflammation. Meantime, it might act as a signal promoter in inflammation. SHP-2 promotes growth factor induced activation of phosphatidylinositol 3-kinase (PI3-K)/Akt, the extracellular signal-related kinases (ERKs) and nuclear factor-kappa B (NF-kB). SHP-2 can either negatively or positively regulate the activation of Janus kinase 2 (Jak2)/signal transducer and activator of transcription (STAT) and the c-Jun-amino terminal kinases (JNKs) depending on different circumstances. rs12423190 may promote the over-expression of SHP-2, further involvement in the up-regulation of inflammatory cytokines through MAPKs and NF- $\mathrm{kB}$ signaling pathways, eventually leading to increase in inflammation related to atrophy [31]. However, the rs2301756 does not appear to reside in transcription factor binding sites or splicing sites, but is in the LD with associated haplotypes. The rs12423190 polymorphism is also in a linkage disequilibrium, which may contain other unidentified causative SNPs. Further studies of PTPN11 sequence variants and their biologic functions may shed light in understanding the association of PTPN11 polymorphisms and the risk of GA.

In addition, we demonstrated that gastric cancer patients had high prevalence of both $H$. pylori seropositivity and gastric atrophy; the prevalence of $H$. pylori seropositivity was significantly higher in subjects with gastric atrophy compared to controls without gastric 
atrophy, implying the important links with of $H$. pylori infection, gastric atrophy, and gastric cancer. Genetic factors, such as PTPN11 polymorphisms may contribute to gastric atrophy, through affecting the connection of Cag A and SHP-2 protein.

The first limitation in our study is the H. pylori infection status being determined by serology, not by the serum CagA antibody test. In East Asia countries, almost all $H$. pylori strains reported from infected patients were East Asian CagA positive strains [32-34], therefore $H$. pylori strains in infected patients most likely possess CagA in our study. The second limitation is the PG criteria for GA screening, the criterion for GA is PGI $<82.3 \mathrm{ng} / \mathrm{ml}$ and PGI/II ratio $<6.05$. These parameters for atrophy are used in China and have been validated in only one histological confirmatory study [23], it is may be insufficient to draw a reliable diagnosis, thus more confirmatory studies are needed. Finally, a small sample size of gastric atrophy, especially for the cohort of $H$. pylori $(+)$ gastric atrophy individuals is due to a low incidence of gastric atrophy in China. A large-scale study for recruiting more patients with gastric atrophy is required to confirm our findings in the future.

\section{Conclusions}

In conclusion, our study provides the first evidence that rs12423190 polymorphism of the PTPN11 gene is significantly associated with an increased risk of gastric atrophy in H. pylori infected Chinese population, suggesting that rs12423190 polymorphism could be used as a biomarker of genetic susceptibility to gastric atrophy.

\section{Competing interests}

No competing interests to be disclosed.

\section{Authors' contributions}

JJ and CX designed and carried out most of study; JZF, KF, JMS WYP and TSY participated data acquisition and analysis; JJ and JZF wrote the first draft of manuscript. All authors contributed to and approved the final manuscript by providing constructive suggestions.

\section{Acknowledgements}

This study has been supported by National Natural Science Foundation of China (30940060 and 81072369). The authors would like to thank everybody participating in this study, particularly to Mr Chang-Song Guo and Ms Ying Song for their technical support.

\section{Author details}

${ }^{1}$ Division of Clinical Epidemiology, Jilin University First Hospital, Changchun, China. ${ }^{2}$ Division of Pathology, Jilin University First Hospital, Changchun, China. ${ }^{3}$ Department of Gastric and Colorectal Surgery, Jilin University First Hospital, Changchun, China.

Received: 19 March 2012 Accepted: 28 June 2012

Published: 12 July 2012

\section{References}

1. Hamiliton SR, Aaltonen L (Eds): WHO classification of tumors. Lyon: IARC Press; 2000.
2. Dai M, Ren JS, Li N, Li Q, Yang L, Chen YH: Estimation and prediction on cancer related incidence and mortality in China, 2008. Zhonghua Liu Xing Bing Xue Za Zhi 2012, 33(1):57-61.

3. Lochhead P, El-Omar EM: Helicobacter pylori infection and gastric cancer. Best Pract Res Clin Gastroenterol 2007, 21(2):281-297.

4. Kabir S: Effect of Helicobacter pylori eradication on incidence of gastric cancer in human and animal models: underlying biochemical and molecular events. Helicobacter 2009, 14(3):159-171.

5. Amieva MR, El-Omar EM: Host-bacterial interactions in Helicobacter pylori infection. Gastroenterology 2008, 134(1):306-323.

6. Snaith A, El-Omar EM: Helicobacter pylori: host genetics and disease outcomes. Expert Rev Gastroenterol Hepatol 2008, 2(4):577-585.

7. Hishida A, Matsuo K, Goto Y, Hamajima N: Genetic predisposition to Helicobacter pylori-induced gastric precancerous conditions. World J Gastrointest Oncol 2010, 2(10):369-379.

8. Kuipers EJ, Perez-Perez Gl, Meuwissen SG, Blaser MJ: Helicobacter pylori and atrophic gastritis: importance of the cagA status. J Natl Cancer Inst 1995, 87(23):1777-1780.

9. Yamazaki S, Yamakawa A, Ito Y, Ohtani M, Higashi H, Hatakeyama M, Azuma $\mathrm{T}$ : The CagA protein of Helicobacter pylori is translocated into epithelial cells and binds to SHP-2 in human gastric mucosa. J Infect Dis 2003, 187(2):334-337.

10. Hatakeyama M: The role of Helicobacter pylori CagA in gastric carcinogenesis. Int J Hematol 2006, 84(4):301-308.

11. Hatakeyama M: Helicobacter pylori and gastric carcinogenesis. J Gastroenterol 2009, 44(4):239-248.

12. Hatakeyama M: Relationship between Helicobacter pylori CagA-SHP-2 interaction and gastric cancer. Nihon Rinsho 2005, 63(Suppl 11):48-52.

13. Chan G, Kalaitzidis D, Neel BG: The tyrosine phosphatase Shp2 (PTPN11) in cancer. Cancer Metastasis Rev 2008, 27(2):179-192.

14. Grossmann KS, Rosario M, Birchmeier C, Birchmeier W: The tyrosine phosphatase Shp2 in development and cancer. Adv Cancer Res 2010, 106:53-89.

15. Jongmans MC, van der Burgt I, Hoogerbrugge PM, Noordam K, Yntema HG, Nillesen WM, Kuiper RP, Ligtenberg MJ, van Kessel AG, van Krieken JH, et al: Cancer risk in patients with Noonan syndrome carrying a PTPN11 mutation. Eur J Hum Genet 2011, 19(8):870-874.

16. Kim JS, Shin OR, Kim HK, Cho YS, An CH, Lim KW, Kim SS: Overexpression of protein phosphatase non-receptor type 11 (PTPN11) in gastric carcinomas. Dig Dis Sci 2010, 55(6):1565-1569.

17. Jamshidi Y, Gooljar SB, Snieder H, Wang X, Ge D, Swaminathan R, Spector TD, O'Dell SD: SHP-2 and PI3-kinase genes PTPN11 and PIK3R1 may influence serum apoB and LDL cholesterol levels in normal women. Atherosclerosis 2007, 194(2):e26-e33.

18. Lu Y, Dolle ME, Imholz S, van 't Slot R, Verschuren WM, Wijmenga C, Feskens EJ, Boer JM: Multiple genetic variants along candidate pathways influence plasma high-density lipoprotein cholesterol concentrations. J Lipid Res 2008, 49(12):2582-2589.

19. Goto Y, Ando T, Yamamoto K, Tamakoshi A, El-Omar E, Goto H, Hamajima $\mathrm{N}$ : Association between serum pepsinogens and polymorphismof PTPN11 encoding SHP-2 among Helicobacter pylori seropositive Japanese. Int J Cancer 2006, 118(1):203-208.

20. Hamajima N, Rahimov B, Malikov Y, Abdiev S, Ahn KS, Bahramov S, Kawai S, Nishio K, Naito M, Goto Y: Associations between a PTPN11 polymorphism and gastric atrophy-opposite in Uzbekistan to that in Japan. Asian Pac J Cancer Prev 2008, 9(2):217-220.

21. Hishida A, Matsuo K, Goto Y, Naito M, Wakai K, Tajima K, Hamajima N: Associations of a PTPN11 G/A polymorphism at intron 3 with Helicobactor pylori seropositivity, gastric atrophy and gastric cancer in Japanese. BMC Gastroenterol 2009, 9:51.

22. Kawai S, Goto Y, Ito LS, Oba-Shinjo SM, Uno M, Shinjo SK, Marie SK, Ishida Y, Nishio K, Naito M, et al: Significant association between PTPN11 polymorphism and gastric atrophy among Japanese Brazilians. Gastric Cancer 2006, 9(4):277-283.

23. Cao Q, Ran ZH, Xiao SD: Screening of atrophic gastritis and gastric cancer by serum pepsinogen, gastrin-17 and Helicobacter pylori immunoglobulin G antibodies. J Dig Dis 2007, 8(1):15-22.

24. De La Vega FM: Selecting single-nucleotide polymorphisms for association studies with SNPbrowser software. Methods Mol Biol 2007, 376:177-193. 
25. Lin DY, Zeng D, Millikan R: Maximum likelihood estimation of haplotype effects and haplotype-environment interactions in association studies. Genet Epidemiol 2005, 29(4):299-312.

26. Zeng D, Lin DY, Avery CL, North KE, Bray MS: Efficient semiparametric estimation of haplotype-disease associations in case-cohort and nested case-control studies. Biostatistics 2006, 7(3):486-502.

27. Zhu F, Loh M, Hill J, Lee S, Koh KX, Lai KW, Salto-Tellez M, lacopetta B, Yeoh $K G$, Soong R: Genetic factors associated with intestinal metaplasia in a high risk Singapore-Chinese population: a cohort study. BMC Gastroenterol 2009, 9:76.

28. Zhang LWP, Liang H, Wen J, Jiang J, Zhang Z, Wei S, Liu C: Correlation of PTPN11 polymorphism at intron 3 with gastric cancer. Med J chin PLA 2011, 36(5):474-477.

29. Zheng H, Alter S, Qu CK: SHP-2 tyrosine phosphatase in human diseases. Int J Clin Exp Med 2009, 2(1):17-25.

30. Higashi H, Tsutsumi R, Muto S, Sugiyama T, Azuma T, Asaka M, Hatakeyama M: SHP-2 tyrosine phosphatase as an intracellular target of Helicobacter pylori CagA protein. Science 2002, 295(5555):683-686.

31. Chong ZZ, Maiese K: The Src homology 2 domain tyrosine phosphatases SHP-1 and SHP-2: diversified control of cell growth, inflammation, and injury. Histol Histopathol 2007, 22(11):1251-1267.

32. Ito Y, Azuma T, Ito S, Miyaji H, Hirai M, Yamazaki Y, Sato F, Kato T, Kohli Y, Kuriyama M: Analysis and typing of the vacA gene from cagA-positive strains of Helicobacter pylori isolated in Japan. J Clin Microbiol 1997, 35(7):1710-1714

33. Miehlke S, Kibler K, Kim JG, Figura N, Small SM, Graham DY, Go MF: Allelic variation in the cagA gene of Helicobacter pylori obtained from Korea compared to the United States. Am J Gastroenterol 1996, 91(7):1322-1325.

34. Pan ZJ, van der Hulst RW, Feller M, Xiao SD, Tytgat GN, Dankert J, van der Ende A: Equally high prevalences of infection with cagA-positive Helicobacter pylori in Chinese patients with peptic ulcer disease and those with chronic gastritis-associated dyspepsia. J Clin Microbiol 1997, 35(6):1344-1347

doi:10.1186/1471-230X-12-89

Cite this article as: Jiang et al:: Association of polymorphism of PTPN 11 encoding SHP-2 with gastric atrophy but not gastric cancer in

Helicobacter pylori seropositive Chinese population. BMC Gastroenterology 2012 12:89.

\section{Submit your next manuscript to BioMed Central and take full advantage of:}

- Convenient online submission

- Thorough peer review

- No space constraints or color figure charges

- Immediate publication on acceptance

- Inclusion in PubMed, CAS, Scopus and Google Scholar

- Research which is freely available for redistribution 